\title{
Case Report \\ Infratentorial single Cystic metastatic lesion: A radiological diagnostic dilemma
}

\author{
${ }^{1}$ Jasiththa A, ${ }^{2}$ Ajantha K
}

\begin{abstract}
Non small cell carcinoma (NSCC) of lung is an unusual cause of single cystic metastatic infratentorial lesion. Infratentorial single cystic lesion is very unusual for metastatic brain varieties though lung carcinoma is the most frequent source of brain metastasis. We reported an uncommon case of a 64 -year - old gentleman presented with cerebellar ataxia. His MRI brain revealed single cystic infratentorial rim enhancing lesion. CT guided biopsy and histopathology favored the diagnosis of metastatic NSCC of lung. According to the radiological findings, there was an ambiguity in differentiating primary from metastatic posterior fossa neoplasm. Therefore, it is pivotal to consider broad differential diagnosis along with histopathological confirmation in identifying single cystic infratentorial brain lesion.
\end{abstract}

\section{Case presentation}

A64-years-old gentleman who had had progressive ataxic gait, vertigo, vomiting and lassitude for two weeks was admitted at the Emergency Unit (EU). He was an Ex-smoker who smoked 10 pack years, five years ago. Clinical examinations showed remarkable cerebellar signs predominantly in lower limbs than upper limbs with abnormal tandem gait.

His routine blood investigations and chest X-ray were normal. Contrast CT brain showed a single space occupying lesion in the posterior fossa. However, MRI revealed an infratentorial, rim enhancing, hypointensed lesion on post contrast T1 weighted image (Figure 1). MR spectroscopy with long echo times (TE) performed at the lesion showed strong peak at creatinine and choline contained compounds (CHO) (Figure 2). His CT (Contrast) Thorax, Abdomen and Pelvis were normal. Bronchoscopic examination revealed a mass lesion at tracheo-bronchial junction (figure 3 ). Histology of both trans-bronchial biopsy and CT guided biopsy of infra tentorial lesion were reported as non small cell carcinoma of lung.

The common differential diagnoses of primary infratentorial single cystic lesion are hemangioblastoma, epidermoid cyst, teratoma, dermoid cyst and craniopharyngioma (extremely rare). Whilst metastatic single infratentorial lesions could be

\footnotetext{
${ }^{1}$ Registrar in Neurology, Teaching Hospital, Jaffna

${ }^{2}$ Consultant Neurologist, Teaching Hospital, Jaffna
}

from lung, breast, malignant melanoma, kidney or gastrointestinal tract ${ }^{1}$. Astrocytoma and medulloblastoma are very uncommon infratentorial neoplastic lesion in adults ${ }^{2,3}$.This patient was referred to oncologist and cardiothoracic surgeon for further evaluation and management.

\section{Discussion}

This patient had major risk factor for lung carcinoma as he had a history of smoking (10 pack years). Therefore, he was investigated initially to rule out Lung malignancy, as a possibly primary lesion, by arranging CXR and CT (contrast) thorax. However, they were inconclusive. Further investigation by MRI spectroscopy showed strong peak at creatinine and choline suggesting a single infratentorial space occupying lesion as a tumor. As the next stage of investigations, the CT guided biopsy and histology of the brain lesion were performed in which features of adenocarcinoma were revealed; but radiological evaluations such as USS and Contrast CT scan of abdomen, pelvis and thyroid did not reveal any abnormalities. Therefore, with a high suspicion of lung malignancy bronchoscopy was carried out; that showed a mass lesion at tracheo- bronchial junction. Further, trans-bronchial biopsy and histology confirmed the diagnosis as NSCC.

The literature survey of the case scenario expressed a wide histopathological spectrum for the radiological diagnosis of cystic intra cranial lesion. However, identifying the location on an imaging would help to narrow down the differential diagnosis. Various hospital-based studies were done in Asian countries reveal brain metastasis outnumbered the primary brain tumors $(10: 1)$ and were more common in males, and lung followed by breast was the most common primary cause leading to metastasis ${ }^{4}$. According to epidemiological data, primary Infratentorial neoplastic lesions are less common than supratentorial in late adulthood $\left(6^{\text {th }}\right.$ decade $)^{4}$. In contrast, single metastatic lesion was identified in less than $30 \%$ of all brain metastasis, and cerebellum was found to be the second common site of metastasis $(15 \%)^{5}$.

Adenocarcinoma, $40 \%$ of NSCC, is the common histopathological subtype identified to produce cystic brain metastasis ${ }^{4}$. Exact mechanism of brain 
cyst formation remains unclear. However, Anaplastic Lymphoma Kinase (ALK) gene rearrangement was described as a possible explanation for the distinct biological abnormality in cystic brain metastasis. ALK related lung cancer has relatively higher tendency to affect younger patients, light smokers or former cigarette smokers. The Echinodermal Microtubule - associated protein - like 4 (EML4) - ALK gene fusion is responsible for majority of adenocarcinoma of lung ${ }^{6}$. Fluorescence In Situ Hybridization (FISH) is the FDA approved kit to detect this gene. Immunohistochemistry (IHC) is also an effective approach to diagnosis of tumors with uncertain

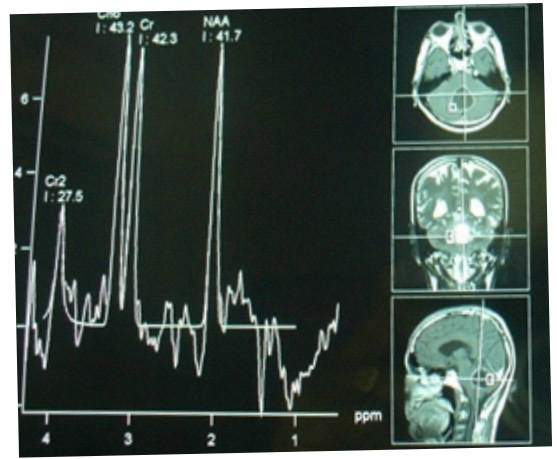

Figure1

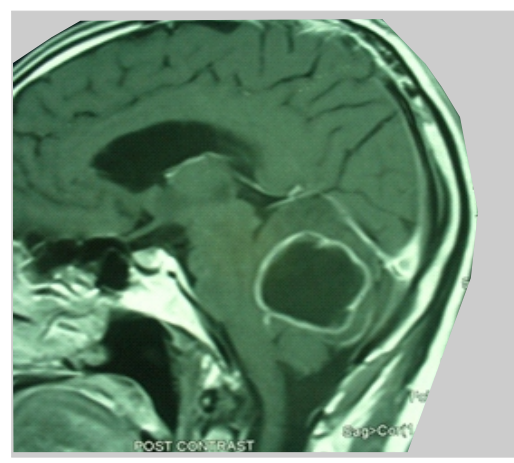

Figure 2 origin, primary and metastatic from unknown primary tumor. However, these tests were not performed in our patient due to unavailability ${ }^{7}$.

\section{Take home message/learning point}

Thus differentiation of these cystic lesion purely based, on various imaging technique may not be a useful tool. IHC armamentarium could be a better tool as it allows for more accurate assessments in cases where primary is radiologically unidentified or in the presence of multiple primary sites ${ }^{8}$.

Figure1: MR spectroscopy with long echo times (TE) showing strong peak at creatinine and choline contained compounds (CHO) at the lesion, Figure2: MRI contrast showing hyperintensed im enhancing lesion in mid posterior fossa, Figure 3: Bronchoscopic view of lung malignancy (semi-oval at trachea-bronchial junction)

\section{References}

1. Barnholtz-Sloan JS, Sloan AE, Davis FG, Vigneau FD, Lai P, Sawaya RE. 2004. Incidence proportions of brain metastases in patients diagnosed (1973 to 2001) in the Metropolitan Detroit Cancer Surveillance System. JClinOncol.22:2865-72.

2. Strauss I, Jonas-Kimchi T, Bokstein F, Blumenthal D, Roth J, Sitt R, Wilson J, Ram Z. 2013. Gliomas of the posterior fossa in adults. $J$ Neurooncol.115(3):401-9.

3. Ostrom QT, Gittleman H, Farah P, Ondracek A, Chen Y, Wolinsky Y, Stroup NE, Kruchko C, Barnholtz- Sloan JS. 2013. CBTRUS statistical report: Primary brain and central nervous system tumors diagnosed in the United States in 2006-2010. Neuro Oncol. 15Suppl 2:ii1-56.

4. Saha, A., Ghosh, S. K., Roy, C., Choudhury, K. B., Chakrabarty, B., \& Sarkar, R. (2013). Demographic and clinical profile of patients with brain metastases: A retrospective study. Asian Journal of Neurosurgery, 8(3), 157-161.

5. Biswas G, Bhagwat R, Khurana R, Menon H, Prasad N, Parikh PM. 2006. Brain metastasisEvidence based management. Journal of Cancer Research and Therapeutics. 2(1)

6. Liao, B.-C., Lin, C.-C., Shih, J.-Y., \& Yang, J. C.-H. 2015. Treating patients with $A L K$ positive non-small cell lung cancer: latest evidence and management strategy. Therapeutic Advances in Medical Oncology, 7(5), 274-290.

7. Duraiyan, J., Govindarajan, R., Kaliyappan, K., \& Palanisamy, M.2012. Applications of immunohistochemistry. Journal of Pharmacy \& Bioallied Sciences, 4(Supp1 2), S307-S309.

8. Pekmezci, M., \& Perry, A. (2013). Neuropathology of brain metastases. Surgical Neurology International, 4(Suppl 4), S245-S255. 